\title{
Peak-to-Average Power Ratio reduction in Wavelet based OFDM using Modified Selective Mapping for Cognitive Radio Applications
}

\author{
Arjun K R* and Surekha T P \\ Department of Electronics \& Communication, Vidyavardhaka College of Engineering, Mysore, India
}

('Corresponding author's e-mail: arjunkrmys@gmail.com)

Received: 22 February 2021, Revised: 22 April 2021, Accepted: 22 May 2021

\begin{abstract}
The prospect of guaranteed quality of service for primary and secondary users in the expression of regulated Peak-to-Average Power Ratio (PAPR) and Bit Error Rate (BER) can be increased in Cognitive Radios using Wavelet based OFDM systems which localize spectrum in realms of time and frequency. However, cutting the Gordian knot of high PAPR associated with the Multi-Carrier Modulation schemes to operate in the linear part of the power amplifier nourishing the balance between PAPR and BER is difficult. In this paper, the phase of the subcarriers is altered by a value of finite, equally spaced phase shifts. The frame of Wavelet-based OFDM (WOFDM) with minimum PAPR guarantees the minimum quality of service (QoS) in terms of Throughput. BER is then identified and transmitted. This modification is applied to various Wavelets and compared amongst WOFDM and FFT-based OFDM schemes. The novelty of this paper lies in using Hadamard and/or Toeplitz matrix to generate phase sequences in a modified SLM technique of Wavelet-based OFDM systems while maintaining the minimum required QoS in Cognitive Radios. The result shows that the proposed technique reduces the PAPR compared to unmodified SLM techniques.
\end{abstract}

Keywords: Cognitive radio, Bit Error Rate (BER), Selective Mapping (SLM), Orthogonal Frequency Division Multiplexing (OFDM), Peak-to-Average Power Ratio (PAPR), Wavelets

\section{Introduction}

In order to appease the need for high data rates within the spectrum scarce environment, Cognitive Radio is used. The Cognitive radio is used to narrate a radio that is apprised of the surrounding around it and can alter its transmission parameter such as transmit power, modulation, etc., according to the interference it sees. With the help of the Multi-Carrier Modulation (MCM), such as FFT-based OFDM or Wavelet-based OFDM, cognitive radios can perceive the available spectrum white space around them. They can also adjust their transmit power, frequencies, modulation schemes, and protocols to access those systems competently and provide service to Secondary Users (SU) in the spectrum allocated initially to Primary Users (PU). When PU is not using the allocated spectrum, the same spectrum can be reallocated to PU when needed [1]. In adapting to the environment and due to in-phase narrow bands used in OFDM systems, the PAPR tends to increase, which will severely affect the system performance as the Power Amplifier can no longer operate in its linear fragment. The primitive requirement of Cognitive Radio is dynamic spectrum access which is fulfilled by OFDM. OFDM provides higher bit rates compared to single carrier transmission [2]. The sinusoid bases function in OFDM has been replaced by Wavelet bases to take advantage of its flexibility and ambidexterity. It also caters to the needs of high data throughput and guaranteed minimum quality of service for both primary users and secondary users in Cognitive Radio Networks [3,4]. 
http://wjst.wu.ac.th

The Wavelet-based OFDM (WOFDM) is an evolving system found in many studies [5-7]. However, the study related to the effects of PAPR in WOFDM for a Cognitive Radio system is limited [8-9]. Figure 1 shows the basic building block of WOFDM for Cognitive Radio, in which the IDFT (Inverse Discrete Fourier Transform) and DFT (Discrete Fourier Transform) blocks of the OFDM system has been replaced by IDWT (Inverse Discrete Wavelet Transform) and DWT (Discrete Wavelet Transform) blocks respectively. The intelligence and adaptability of Cognitive Radio lie mainly in feedback from channel to spectrum sensing block, which decides to choose the right modulation, coding, Wavelet-basis, and configures radio according to the requirement laid by the service provider.

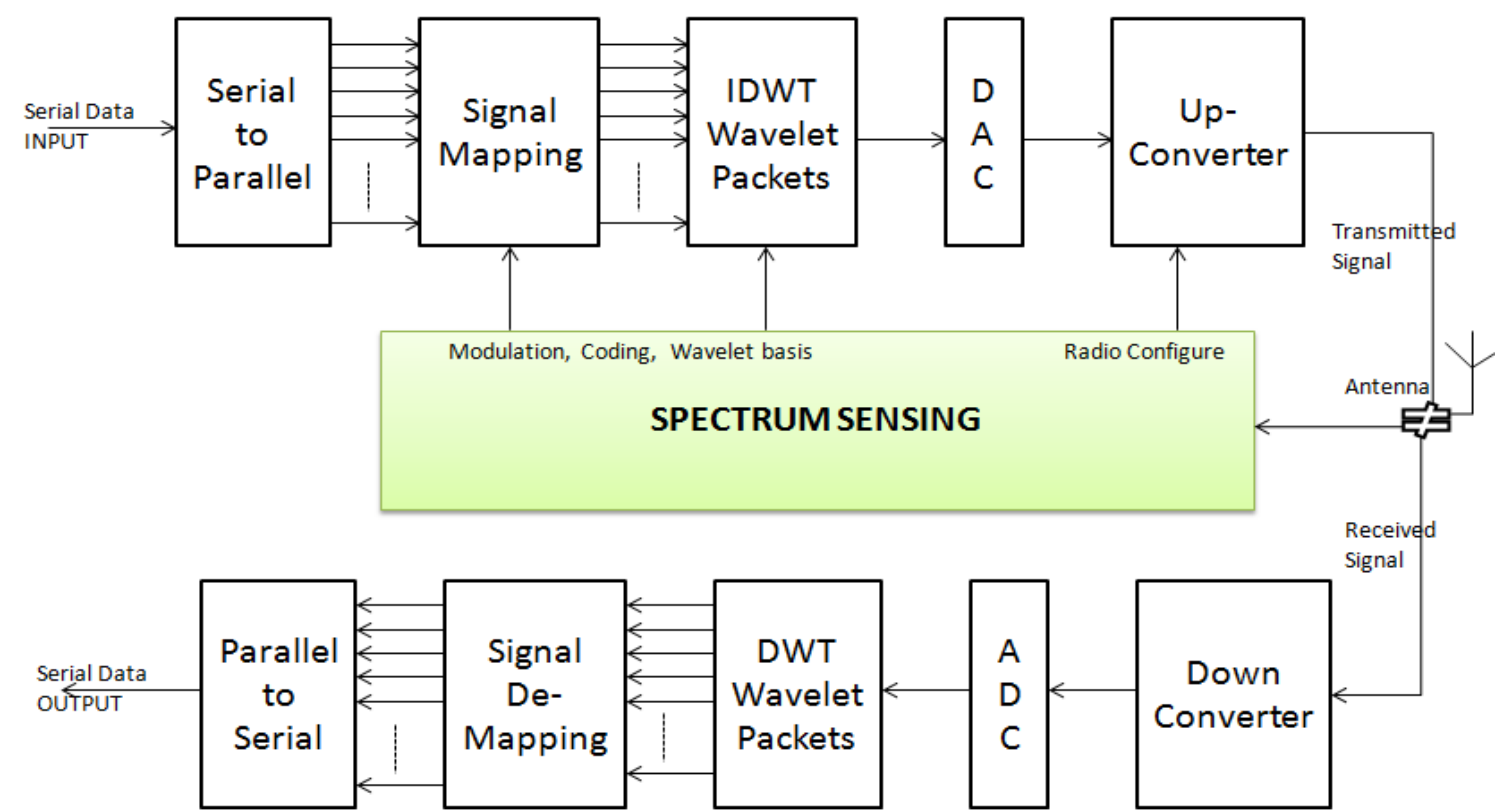

Figure 1 Basic building block of Wavelet based OFDM for Cognitive Radio Systems.

In this paper, a study on the PAPR performance of conventional OFDM and Wavelet-based OFDM is presented. The dissimilitude of BER with different Wavelets is ascertained. The signal characteristics of various Wavelets like Haar, Daubechies, Symlets, Coiflets, and Biorthogonal with varying filter length on PAPR with varying number of subcarrier is investigated with modified phase orthogonal matrix SLM, and conventional SLM is presented. The remainder of the study is structured as follows. A brief overview of OFDM and WOFDM is presented in sections 2 and 3, respectively. An overview of PAPR and PAPR reduction techniques is presented in sections 4 and 5, followed by; Quality of service in Cognitive Radio for spectrum sensing is presented in section 6. However, Spectrum Sensing is out of the scope of this paper. A modified SLM scheme with the algorithm is proposed and presented with simulation results in sections 7 and 8 . Finally, in section 9, the paper is concluded.

\section{Orthogonal Frequency Division Multiplexing (OFDM)}

OFDM consists of conventional modulators and demodulators, each with different orthogonal carrier frequencies, and is a form of multi-carrier modulation technique. A combination of modulator outputs with orthogonal subcarriers would comprise the transmitter output. Let us take into account the transmission of $\mathrm{N}$ data defined as, $\mathrm{A}_{\mathrm{k}}, \mathrm{k}=0,1, \ldots, \mathrm{N}-1$ where $\mathrm{A}_{\mathrm{k}}$ is a complex output of modulators such as QPSK or QAM. Suppose that the frequency of the $k^{\text {th }}$ carrier for $X_{k}$ is $f_{k}$. Then, the output from the transmitter is given by, 
http://wjst.wu.ac.th

$a(t)=\sum_{k=0}^{N-1} A_{k} e^{j 2 \pi f_{k} t}$

A digital source in a sampled-data fashion will generate its output. The digital MCM transmitter output will be as given in Eq. (2) by allowing $\mathrm{t}=n T_{S}$, where $T_{S}$ is the sample interval,

$a\left(n T_{s}\right)=\sum_{k=0}^{N-1} A_{k} e^{j 2 \pi f_{k} n T_{s}}$

The output of the MCM transmitter in Eq. (2) takes the form of Eq. (3) with frequency spacing $f_{s}$, i.e.

$$
\begin{aligned}
& f_{k}=\mathrm{k} f_{s}, \mathrm{k}=0,1, \ldots, \mathrm{N}-1, \\
& a\left(n T_{s}\right)=\sum_{k=0}^{N-1} A_{k} e^{j 2 \pi \mathrm{k} f_{s} n T_{s}}
\end{aligned}
$$

Letting $f_{s}=1 / \mathrm{N} T_{s}$ which is the minimum parting between the signals of different modulators to preserve accurate angularity. The OFDM output signal corresponding to this is given by,

$a_{n}=a\left(n T_{S}\right)=\sum_{k=0}^{N-1} A_{k} e^{j 2 \pi n \mathrm{k} / \mathrm{N}}$

The Spectral efficiency of OFDM is relatively high for the higher number of subcarriers and Filter Bank based Multi Carrier (FBMC) is suitable for a limited number of subcarriers where the spectral efficiency is comparatively greater than that of OFDM [7].

\section{Wavelet based OFDM (WOFDM)}

WOFDM, derived from multi-resolution analysis, is implemented using orthogonal Wavelet packet bases. The major advantage of WOFDM is the absence of Cyclic Prefix which consumes nearly $20 \%$ of bandwidth in conventional OFDM systems. The feature of the orthogonal bases is derived from a quadrature mirror filter (QMF) pair consisting of a high-pass filter $\mathrm{g}[\mathrm{n}]$ and a low-pass filter $\mathrm{h}[\mathrm{n}]$, each with a length of L. Such filters are identified by [6]:

$g[L-1-n]=(-1)^{n} h[n]$

Such filters have duals that are flipped variants of complex conjugate time given as:

$h^{\prime}[n]=h^{*}[-n]$ and $g^{\prime}[n]=g^{*}[-n]$

The filter pairs in Eq. (6) are referred to as synthesis filters and are used in the transmitter to produce Wavelet packets. From these filters, the bases of the Wavelet packets are given as:

$$
\begin{aligned}
& \xi_{l+1}^{2 p}=\sqrt{2} \sum_{k} h[k] \xi_{l}^{p}[2 n-k] \\
& \xi_{l+1}^{2 p+1}=\sqrt{2} \sum_{k} g[k] \xi_{l}^{p}[2 n-k]
\end{aligned}
$$


where, p in Eq. (7) is subcarrier index, $\xi$ denotes Wavelet packet duals and the number of subcarriers is given as $N=2^{l}$ and 1 is the number of decomposition levels in the filter banks. The WOFDM output is referred to as the Inverse Discrete Wavelet Packet Transform (IDWPT), analogous to traditional OFDM Inverse Fourier transforms. Correspondingly, the WOFDM is given by:

$S[n]=\sum_{u} \sum_{k=0}^{N-1} a_{u, k} \xi_{l}^{k}[n-u N]$

where ' $\mathrm{k}$ ' in Eq. (8) denotes the index of subcarriers and ' $u$ ' denotes the index of WPM symbols.

\section{Peak-to-Average Power Ratio (PAPR)}

The number of single modulated sub-carriers in a multi-carrier signal contributes greatly to a relatively broad peak to average power ratio as they repeatedly add up coherently. The PAPR of Eq. (4) or Eq. (8) is given as:

$P A P R=\frac{\max _{0 \leq n \leq M-1}(|x[n]|)^{2}}{E(|x[n]|)^{2}}$

where, $E(||$.$) , in Eq. (9) is the anticipated value and denotes the average.$

Cumulative Distributive function (CDF) is one of the most frequently used performance measures for PAPR reduction technique. The $\mathrm{CDF}$ of the power is given as:

$F(z)=\int_{0}^{z} \frac{1}{2 \sigma^{2}} e^{-\frac{u}{2 \sigma^{2}}} d u=1-e^{-\frac{z}{2 \sigma^{2}}}$

Eq. (10) is obtained by considering the fact of central limit theorem for sizeable number of subcarriers $\mathrm{M}$, the multi carrier components follow the Gaussian distribution, each with zero mean and variance of $\mathrm{M}$ times the variance of 1 complex sinusoid. In this paper Complementary CDF (CCDF) is used as performance parameter, where CCDF is the probability that PAPR is below some threshold level $\mathrm{z}$, can be written as,

$\operatorname{PAPR}(M, z)=\operatorname{Pr}\{P A P R \leq z\}=[F(z)]^{M}=1-\left(1-\left(1-e^{-P A P R}\right)\right)^{M}$

where $\operatorname{Pr}\{\mathrm{x}\}$ is the probability of occurrence of the case $\mathrm{x}$.

The PAPR of WOFDM is slightly difference than OFDM. The PAPR in OFDM is calculated per symbol; whereas, PAPR in WOFDM is calculated per frame because of the fact that symbols in WOFDM overlap in the time domain. The PAPR per frame of WOFDM in dB is given as [8],

$\mathrm{PAPR}=10 \log _{10} \frac{\max \left(x_{t} \times \operatorname{conj}\left(x_{t}\right)\right)}{E\left(x_{t} \times \operatorname{conj}\left(x_{t}\right)\right.}$

$x_{t}$ in Eq. (12) denotes the WOFDM symbol with $\mathrm{N}$ carriers and $\mathrm{N}$ symbols per frame. Max, conj and $\{\mathrm{E}\}$ represents maxima operator, conjugate operator and average respectively. 
http://wjst.wu.ac.th

\section{PAPR Reduction techniques}

Various types of PAPR reduction techniques are presented in the literature. PAPR reduction techniques were categorized into signal distortion and signal scrambling techniques by Torun et al. [11]. Sandoval et al. [10] suggested the division into 4 groups of PAPR reduction techniques, namely: coding schemes including (convolutional coding, Block coding, concatenate coding), Multiple Signaling \& Probabilistic (MSP) which includes Partial Transmit Sequence (PTS), selective mapping (SLM), Interleaving etcetera. Techniques such as clipping and filtering, peak windowing, peak cancellation and companding with hybrid PAPR reduction techniques are used and are known as signal distortion (SD) techniques. However, in this paper we have considered SLM technique which possesses an advantage of low complexity [12] and an ease in the implementation.

\section{Conventional Selective Mapping (SLM)}

Conventional Selective Mapping (SLM) is a popular technique towards PAPR reduction, and it is often used in multi-carrier modulation schemes. Although conventional SLM significantly reduces the PAPR, it generates phase sequence randomly, and it lacks systematic structure, which increases complexity in implementation. Liang [13] proposed an SLM technique in which the structure of ReedMuller codes is used to generate phase sequences. In the above study, both conventional and modified SLM methods are compared, and simulation results claim superior performance of modified SLM compared to Conventional SLM techniques. The additional side information transmission requirement of SLM is addressed in a paper proposed by Valluri et al. [14], in which a simple SI cancellation algorithm using channel estimation-related pilots is proposed. The author presented a revamped cluster architecture that ensures that each cluster has at least one pilot. The above technique is implemented in Universal Software Radio Peripheral (USRP), indicating a significant reduction in PAPR without affecting the signal to noise ratio performance.

Sudha et al. [15] proposed 3 low complexity SLM techniques to reduce PAPR, out of which in the $1^{\text {st }}$ technique $\mu$-law companding is employed with a low complexity SLM generating ' $v$ ' number of candidate signal using only 1 IFFT block. Two IFFT blocks are used in scheme two and in $3^{\text {rd }}$ method $(\mathrm{v} / 2+1)$ IFFT blocks are used. The author compared all 3 schemes with conventional SLM and claims that the $1^{\text {st }}$ scheme performs better than the rest of the techniques.

\section{Modified Selective Mapping (SLM)}

The increased number of phase sequences in SLM increases the complexity of IFFT. Singal and Kedia [16] proposed 2 modified SLM techniques in which the complexity of conventional SLM can be reduced by generating alternative signal sequences. This alternative uses the combination of SLM with additive mapping and the latter is generated using $U^{2}$ phase sequences. Finally, the computational complexity of both the modified SLM techniques has been compared with the combination of Additive mapping and $U^{2}$ phase sequences and found that the $3^{\text {rd }}$ technique yields better PAPR performance at the cost of extra memory elements.

Deepa and Kumar [17] have proposed an updated T-transform based SLM to decrease the computational complexity of traditional SLM. The updates are applied to T-transform by dividing and and conquering the approach to large size $\mathrm{N}$ into smaller chunks of $\mathrm{N}$. This modified technique is applied to OFDM system specified in IEEE802.16d-2004 standards.

Bharati and Podder [18] proposed a hybrid method combining clipping and SLM. The author has compared both the techniques for OFDM scheme and found that the fusion technique performs better compared to conventional SLM, which has relatively more energy efficiency compared to single method.

\section{QoS in Cognitive Radio}

The primary function of the Cognitive Radio system is to identify the spectrum holes. The process of identifying spectrum holes may directly impact on QoS. QoS may vary depending on the application in Cognitive Radio Network. Giweli et al. [19] claims that the delay involved in spectrum sensing from 1 
http://wjst.wu.ac.th

method to other even under similar channel conditions may vary and has greater impact on QoS in terms of throughput. The authors [20] have presented different spectrum sensing techniques and their adequacies in spectrum sharing in 5G RANGE. It has been introduced and the Transmit Power of Primary User (Licensed User) is one of the key parameters in increasing the probability of detection 'Pd' and in the considerations of various channel conditions with different Transmit power threshold.

Spectrum sensing based on energy is one of the Cognitive Radio's simplest and most commonly used techniques. Arjoune et al. [21] investigated a dynamic threshold technique to overcome the drawback of blind energy detection, in which the detection simply cannot differentiate between noise and signal. The proposed method has been implemented on USRP and GNU radios.

Plata and Reátiga [22] proposed the threshold selection process in energy-based spectrum sensing using a method known as Constant False Alarm Rate (CFAR) based on conditions of present noise levels. The simulation results show the increase in probability of detection under dynamic threshold levels compared to constant threshold.

\section{Proposed methodology}

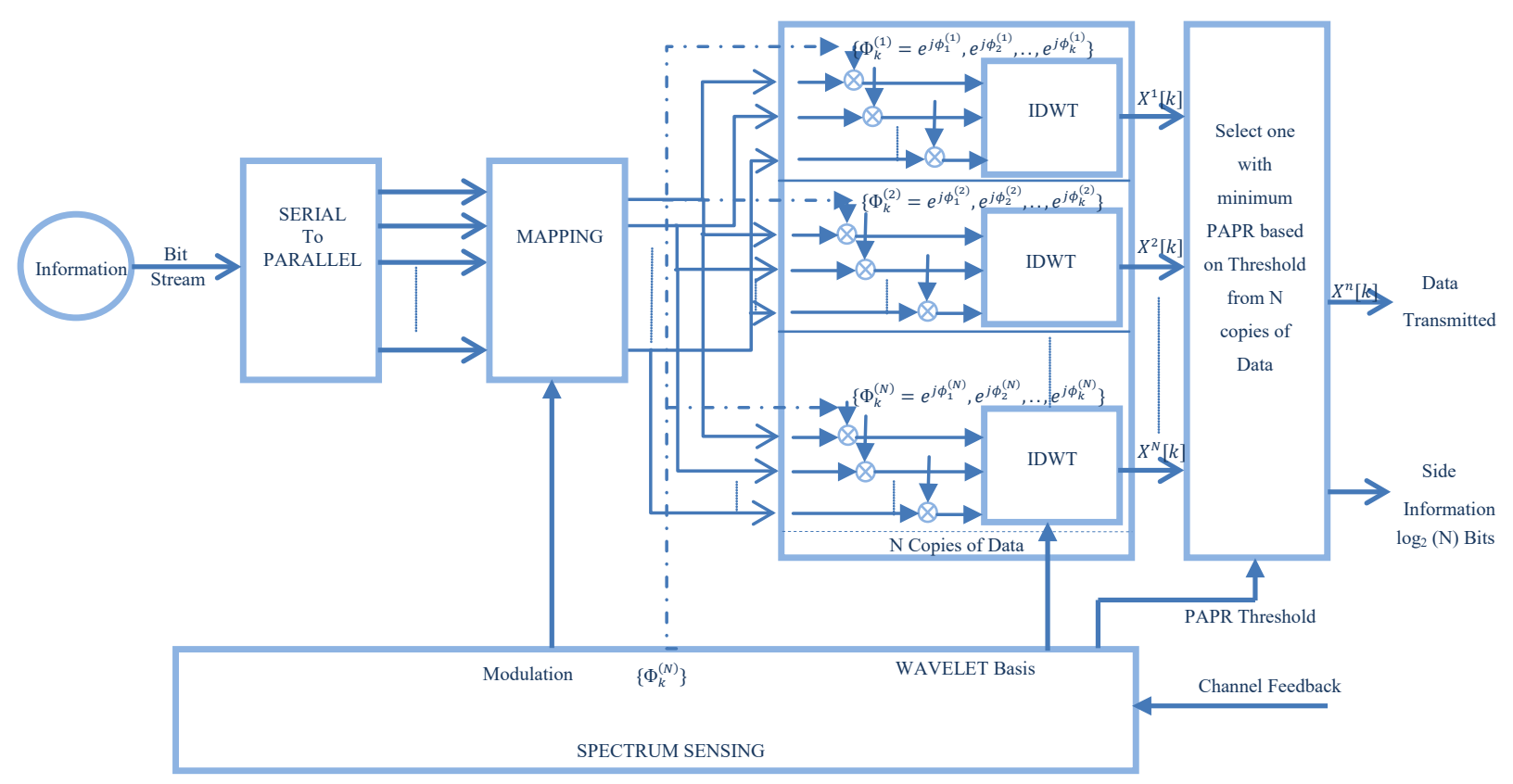

Figure 2 WOFDM transmitter with modified SLM.

From the literature, it is evident that SLM technique with additional side information provides good PAPR reduction. However, in Cognitive Radio Networks, the transmitted power after PAPR reduction from PU will be very low which is barely detectable by the SU. Eventually, this leads to the increase in probability of False Detection $\left(\mathrm{P}_{\mathrm{fd}}\right)$ or no detection of spectrum white space and results in reduction of QoS provided to SU. In order to overcome the problem of the increase in $\mathrm{P}_{\mathrm{fd}}$ and to provide guaranteed QoS in Cognitive Radio (CR) system for SU and to reduce PAPR, we propose a modified SLM technique with additional threshold power as an input.

Figure 2 shows the block diagram of proposed WOFDM transmitter scheme with PAPR reduction while maintaining the QoS. The Information source is $1^{\text {st }}$ converted from serial to parallel and the length of parallel stream is dependent on the constellation stream which is decided by modulation selection block (16/32 qam). The output of constellation mapper will have finite ' $\mathrm{N}$ ' copies replicated. Each copies 
http://wjst.wu.ac.th

of the data stream will be phase shifted by phase vector $\left\{\Phi_{k}^{(n)}\right\}$ generated using orthogonal n X k matrix. Where $\mathrm{N}$ is the frame number and $\mathrm{K}$ is the subcarrier index. The WOFDM symbol after phase shift is given as,

$X^{n}(k)=X(k) \times \Phi_{k}^{(n)}=X(k) \times \mathrm{e}_{k}^{j \emptyset_{k}^{(n)}}$

The phase modified sequences shown in Eq. (13) will be converted into time domain (IDWT) using Wavelet basis function selected depending upon feedback from channel. The lowest PAPR which is greater than or equal to $P_{\min }$ will be selected out of $\mathrm{N}$ phase shifted copies of WOFDM frame.

The algorithm to calculate minimum PAPR while ensuring minimum QoS is given below,

1) Convert information to parallel depending on the selected constellation stream.

2) Clone the data finite number of times. ( $\mathrm{N}$ times in this case).

3) Generate phase sequences using orthogonal matrix with the size $n \mathrm{X} \mathrm{k}$ using Hadamard or Toeplitz matrixes.

4) Multiply each cloned data frame with unique row of phase shifted sequence (subcarrier wise).

5) Convert all frames to time domain (IDWT operation $\left.=x=\operatorname{IDWT}\left(x^{n}[k]\right)\right)$

6) Calculate and list all PAPR using Eq. (12) for all copies of time domain signals.

7) Obtain the minimum power threshold $P_{\text {min, }}$ to ensure user visibility to CR system. Eg. -5 or $2 \mathrm{db}$.

8) Discard PAPRs with powers less than $P_{\min }$.

9) Select the frame with minimum PAPR in the remaining copies of signal, shown in below equation.

where $P A P R p \min \left(x^{n}\right)$ is the PAPR of signals greater than or equal to $\mathrm{P}_{\min }$.

$\hat{x}=\arg \min _{1 \leq n \leq N}\left(P A P R \operatorname{Rin}\left(x^{n}\right)\right)$

Send the index number of selected PAPR frame as a side information to invert and reproduce at the receiver.

\section{Simulation results}

In this section, we begin with the selection and comparison of Wavelets and its length with OFDM without applying PAPR reduction techniques. This is followed reporting the PAPR performance of the modified SLM technique with Hadamard and Toeplitz matrix. The selection of Wavelet is based on BER, Peak-to-Average Power ratio and the transmit power. All simulations are carried out using configuration shown in Table 1.

Table 1 Simulation parameters.

\begin{tabular}{|c|c|c|}
\hline Specifications & OFDM & WOFDM \\
\hline No. of Subcarriers & 256 & 256 \\
\hline Modulation Order-QAM & 4 & 4 \\
\hline Subcarrier Spacing & $15 \mathrm{kHz}$ & $15 \mathrm{kHz}$ \\
\hline Carrier Frequency & $2.5 \mathrm{gHz}$ & $2.5 \mathrm{gHz}$ \\
\hline No. of Symbols & 14 & 20 \\
\hline Cyclic Prefix length & $4.76 \mu \mathrm{s}$ & -nil- \\
\hline Min PAPR for Secondary User- $\mathrm{P}_{\min }$ & $-\mathrm{NA}-$ & $5 \mathrm{~dB}$ \\
\hline
\end{tabular}


http://wjst.wu.ac.th

Figure 3 shows the BER vs SNR performance of Wavelet OFDM and FFT based OFDM. WOFDM with Dabuchies20 attains similar BER of FFT-OFDM at nearly $6 \mathrm{~dB}$ less SNR. CCDF plot in Figure 4 shows the performance of FFT-OFDM and WOFDM with various Wavelet bases. It is evident from the plot that FFT-OFDM and WOFDM with Dabuchies Wavelet filter of 2, 10, 15 and 20 exhibits similar PAPR performance without using any PAPR reduction techniques. Figure 5 shows the normalized transmit power of various Wavelet bases. Various Wavelet bases like Dabuchies2, Dabuchies20, Coiflet10, Symlet30, Biorthogonal and Haar Wavelets are used and found 20 exhibits comparatively less amplitude variations than other Wavelet bases.

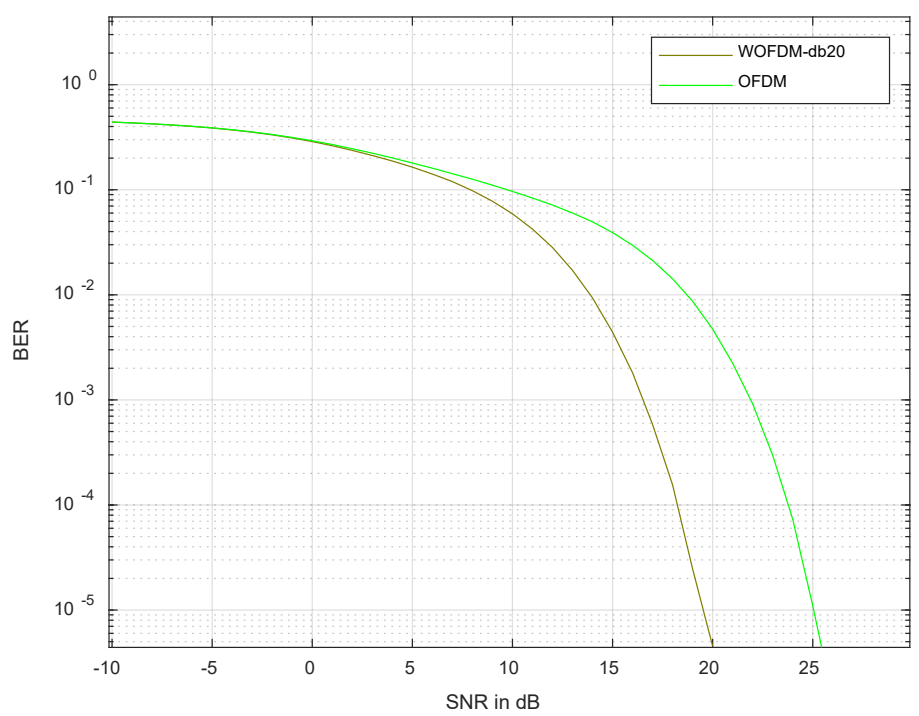

Figure 3 BER vs SNR plot of WOFDM (db-20) and OFDM.

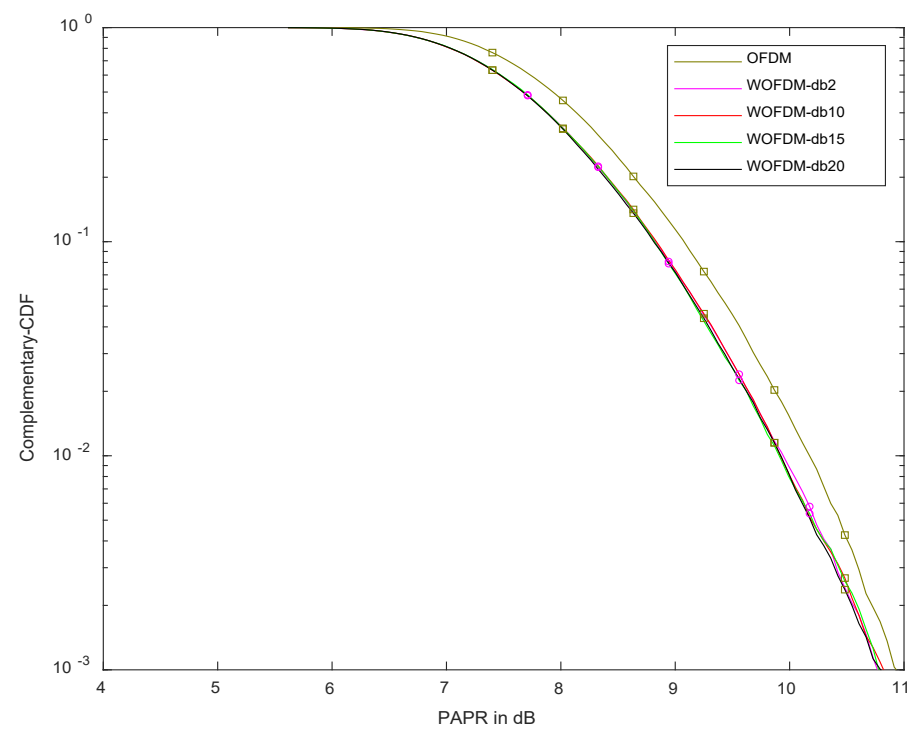

Figure 4 CCDF plots of PAPR in $\mathrm{dB}$ of OFDM and WOFDM. 


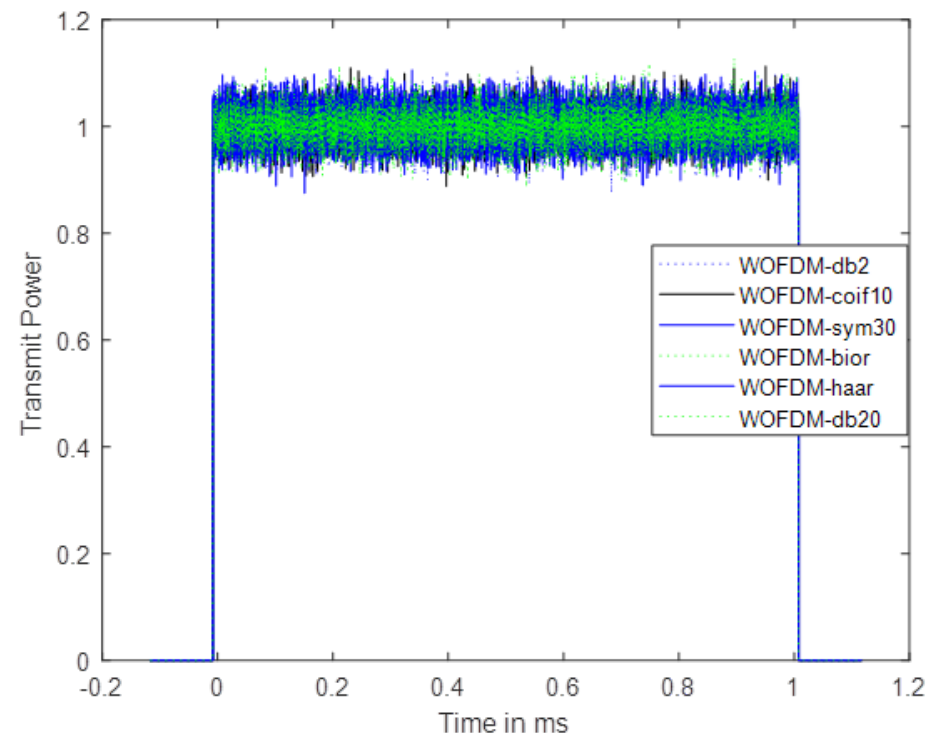

Figure 5 Normalized transmit power of WOFDM with various Wavelet bases.

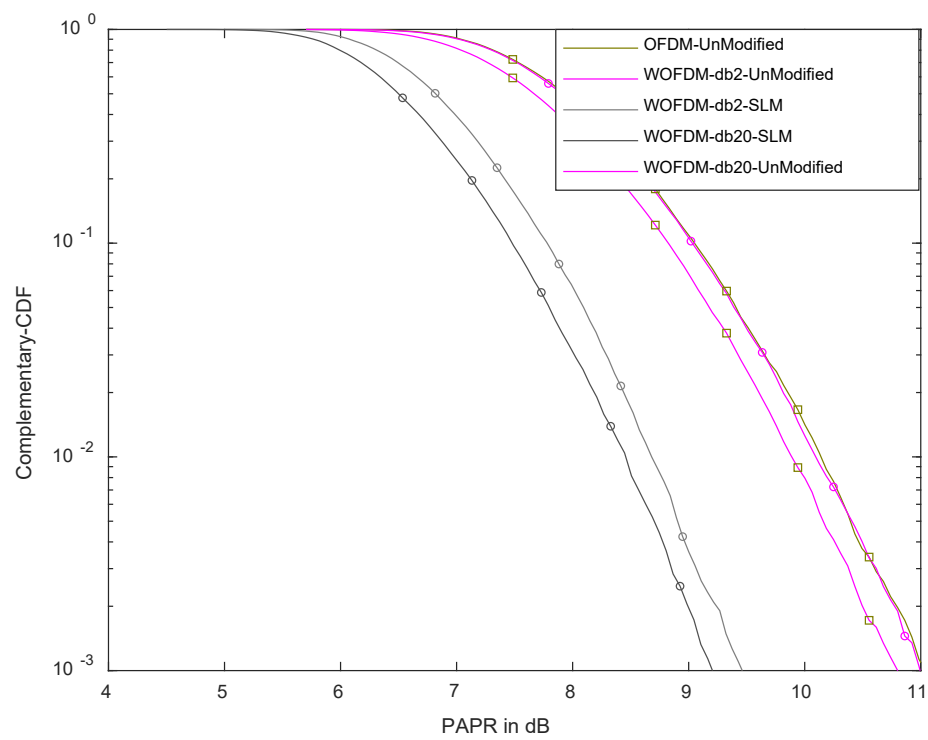

Figure 6 Comparision of prososed SLM scheme with unmodified OFDM and WOFDM with db2 and db20 Wavelet bases. 


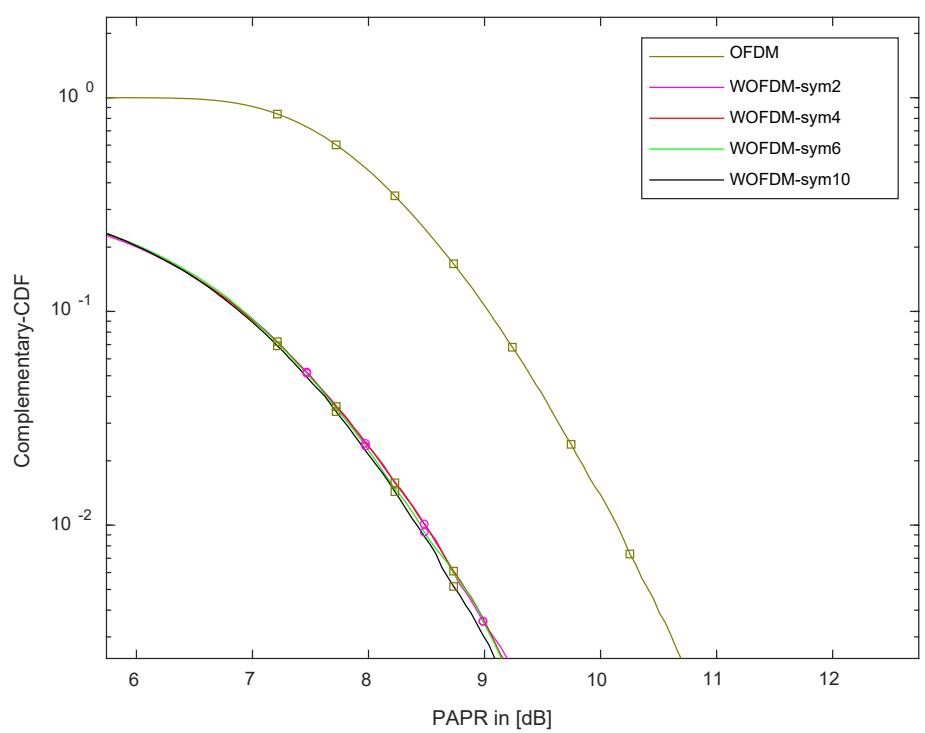

Figure 7 Comparison of proposed SLM scheme with phase shift generated using Hadamard Matrix for symlet Wavelet bases with different filter length.

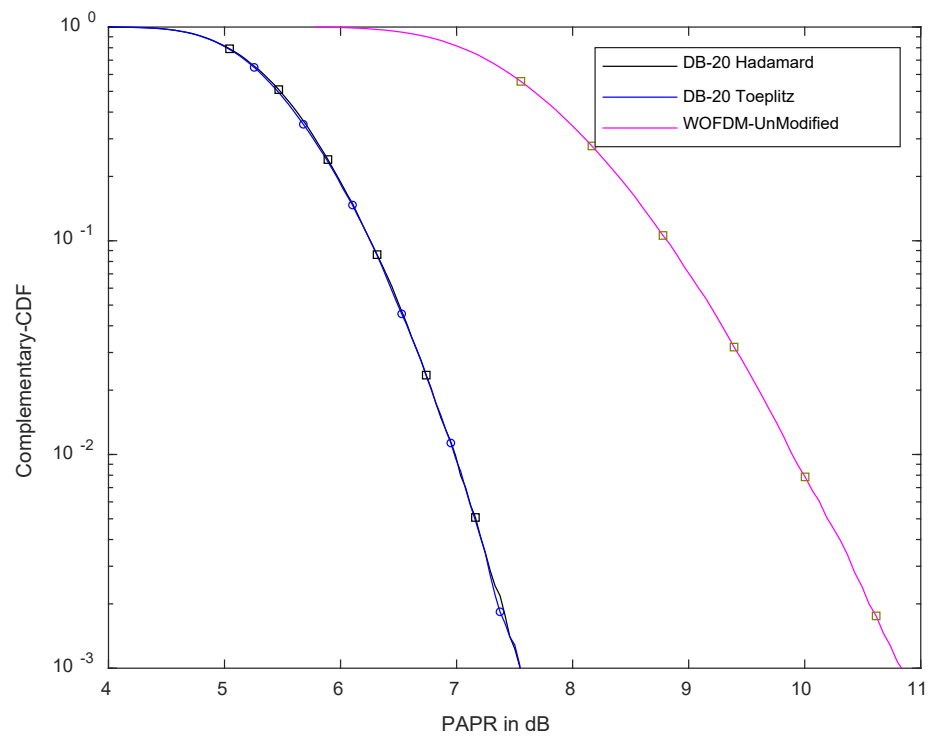

Figure 8 Comparison of proposed SLM scheme with Hadamard and Toeplitz Matrix for db20 Wavelet bases.

From Figure 3 it is evident that WOFDM achieves similar BER to that of OFDM at nearly $6 \mathrm{~dB}$ less power. Since Cyclic Prefix is not required in WOFDM, the bandwidth of WOFDM can be increased by $25 \%$ compared to OFDM. Figure 4 shows the CCDF of unmodified SLM OFDM and WOFDM with various length Wavelet filters and shows almost similar results compared to OFDM and WOFDM. 
http://wjst.wu.ac.th

Figure 5 shows normalized transmit power of WOFDM with various Wavelet bases in which the total variations of transmit power is restricted to 0.4 units of transmit power. Figure 6 shows the CCDF plots of proposed SLM technique using Hadamard matrix with phase of $\{0, \pi / 2, \pi$, and $3 \pi / 2\}$ is used with the threshold $\mathrm{P}_{\min }$ of $5 \mathrm{db}$ is used. Figure 7 shows the simulation result comparing CCDF of OFDM with WOFDM symlet Wavelet with different filter sizes. Figure 8 shows the similar configuration of Figure 6 except with the phase modification of $\{\pi / 4,3 \pi / 4,5 \pi / 4$, and $7 \pi / 4\}$ with Hadamard and Toeplitz matrix which exhibits same PAPR performance. However, with phase shift of $\{\pi / 4,3 \pi / 4,5 \pi / 4$, and $7 \pi / 4\}$ results in improved PAPR performance by $3 \mathrm{~dB}$.

\section{Conclusions}

The paper addresses 2 problems. One is to ensure minimum transmit power required to increase channel capacity, which can lead to an increase in the probability of detection of Primary users and can ensure the minimum Quality of Service. Second is to reduce the PAPR by changing the phase of the WOFDM subcarriers by generating phase shifts using either Hadamard or Toeplitz matrix. It is evident from the simulation results that $\mathrm{db} 20$ performs better than other Wavelet bases. Thus, it can be suggested that it is suitable for the simulated parameters of Table 1 which is similar to LTE. PAPR of unmodified OFDM and WOFDM are similar. Using the proposed methodology, the PAPR is reduced by $3 \mathrm{~dB}$ and Wavelet based OFDM outperforms conventional OFDM (FFT based). The modified SLM with additional side information and computation overhead exhibits improved PAPR performance.

\section{References}

[1] E Biglieri, A Goldsmith, L Greenstein, N Mandayam and H Poor. The concept of cognitive radio. In: Principles of cognitive radio. Cambridge University Press, Cambridge, 2012, p. 1-40.

[2] I Budiarjo, H Nikookar and LP Ligthart. Cognitive radio modulation techniques. IEEE Signal Process. Mag. 2008; 25, 24-34.

[3] H Nikookar. Wavelet radio: Adaptive and reconfigurable wireless systems based on wavelets. Cambridge University Press, Cambridge, 2013.

[4] NT Le, SD Muruganathan and AB Sesay. Peak-to-Average Power Ratio reduction for wavelet packet modulation schemes via basis function design. In: M Almeida (Ed.). Vehicular technologies: Increasing connectivity. Intech Open, 2011.

[5] S Sarowa, N Kumar, S Agrawal and BS Sohi. Evolution of PAPR reduction techniques: A wavelet based OFDM approach. Wireless Pers. Commun. 2020; 115, 1565-88.

[6] D Karamehmedovic, MK Lakshmanan and H Nikookar. Performance of wavelet packet modulation and OFDM in the presence of carrier frequency and phase noise. In: Proceedings of the 2008 European Conference on Wireless Technology, Amsterdam, Netherlands. 2008, p. 166-9.

[7] R Nissel, S Schwarz and M Rupp. Filter bank multicarrier modulation schemes for future mobile communications. IEEE J. Sel. Areas Commun. 2017; 35, 1768-82.

[8] G Kaddoum. Design and performance analysis of a multiuser OFDM based differential chaos shift keying communication system. IEEE Trans. Commun. 2016; 64, 249-60.

[9] MS Iqbal, S Hussain and A Ghafoor. Peak to average power ratio based spatial spectrum sensing for cognitive radio systems. Comput. Electr. Eng. 2017; 63, 30-40.

[10] F Sandoval, G Poitau and F Gagnon. Hybrid Peak-to-Average Power Ratio reduction techniques: Review and performance comparison. IEEE Access. 2017; 5, 27145-61.

[11] B Torun, MK Lakshmanan and H Nikookar. Peak-to-Average Power Ratio reduction of wavelet packet modulation by adaptive phase selection. In: Proceedings of the $21^{\text {st }}$ Annual IEEE International Symposium on Personal, Indoor and Mobile Radio Communications, Istanbul, Turkey. 2010, p. 105-10.

[12] CP Li, SH Wang and CL Wang. Novel low-complexity SLM schemes for PAPR reduction in OFDM systems. IEEE Trans. Signal Process. 2010; 58, 2916-21. 
http://wjst.wu.ac.th

[13] HY Liang. Selective mapping technique based on an adaptive phase-generation mechanism to reduce peak-to-average power ratio in orthogonal frequency division multiplexing systems. IEEE Access 2019; 7, 96712-8.

[14] SP Valluri, V Kishore and VM Vakamulla. A new selective mapping scheme for visible light systems. IEEE Access. 2020; 8, 18087-96.

[15] V Sudha, M Syamkumar and DS Kumar. A low complexity modified SLM and companding based PAPR reduction in localized OFDMA. Wireless Pers. Commun. 2017; 96, 3207-26.

[16] A Singal and D Kedia. Performance analysis of MIMO-OFDM system using SLM with additive mapping and $U^{2}$ phase sequence for PAPR reduction. Wireless Pers. Commun. 2020; 111, 1377-90.

[17] T Deepa and R Kumar. Performance evaluation of a low complexity row-column transform approach for SLM based OFDM transmission system. Wireless Pers. Commun. 2016; 87, 1357-69.

[18] S Bharati and P Podder. Adaptive PAPR reduction scheme for OFDM using SLM with the fusion of proposed clipping and filtering technique in order to diminish PAPR and signal distortion. Wireless Pers. Commun. 2020; 113, 2271-88.

[19] N Giweli, S Shahrestani and HS Cheung. Spectrum sensing in Cognitive Radio Networks: QoS considerations. In: Proceedings of the $7^{\text {th }}$ International Conference on Networks and Communications, Sydney, Australia. 2015.

[20] 5GRANGE. D4.2 - Spectrum Sensing to Complement Databases. 2018.

[21] Y Arjoune, ZE Mrabet, HE Ghazi and A Tamtaoui. Spectrum sensing: Enhanced energy detection technique based on noise measurement. In: Proceedings of the 2018 IEEE $8^{\text {th }}$ Annual Computing and Communication Workshop and Conference, Las Vegas, Nevada. 2018, p. 828-34.

[22] DMM Plata and ÁGA Reátiga. Evaluation of energy detection for spectrum sensing based on the dynamic selection of detection-threshold. Proc. Eng. 2012; 35, 135-43. 